\title{
A generalized rate-dependent constitutive law for elastomeric
}

\section{bearings}

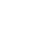

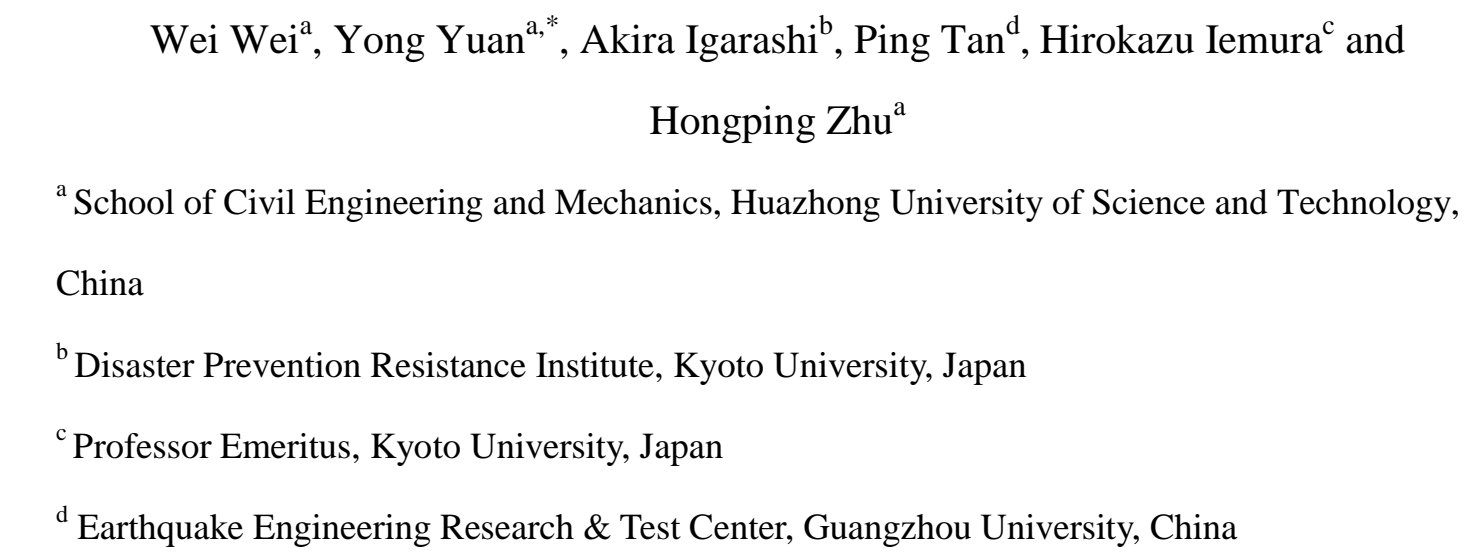

\section{ABSTRACT}

Elastomeric bearings have been a mature and efficient technique to mitigate structural damage caused by earthquakes. This paper proposed a generalized mathematical model for accurately evaluating the rate-dependent stress-strain response of natural rubber bearing (NRB), high damping rubber bearing (HDRB) and super high damping rubber bearing (SHDRB). A novel strain energy function including an additional stiffness correction factor $\alpha$ is proposed to describe the Fletcher-Gent effect of elastomeric materials, which featured by high initial stiffness at small strain ranges. The proposed constitutive model is composed of two parts, the first part consisting of a hyperelastic spring represents the rate-independent equilibrium stress, while the second part consisting of a Maxwell element expresses the rate-dependent overstress.

2 Parameter identification scheme is implemented based on the results of the multi-step 3 relaxation tests and monotonic shear tests. Numerical simulations for monotonic shear

* Corresponding author at: School of Civil Engineering \& Mechanics, Huazhong University of Science \&Technology, Wuhan, China. Tel.:+86 27 87544394; Fax: +86 2787542231.

E-mail address: yuanyong@ @ust.edu.cn (Y. Yuan). 
tests were conducted to demonstrate the capacity of the proposed model in predicting the stress-strain relationship of elastomeric bearings at different strain rates. Finally, in order to investigate the accuracy and feasibility of the proposed model on the application to the seismic response assessment of isolated bridges, real-time hybrid simulation (RTHS) test was performed and favorable results were obtained.

Keywords: Elastomeric bearing; Constitutive law; Rate-dependency; Seismic response

\section{Introduction} buildings and bridges from earthquakes for both new constructions and retrofit projects, and have proven to be effective and relaible. Among various types of elastomeric bearings, natural rubber bearing (NRB) and lead rubber bearing (LRB) have been well known and the application of them in civil structures has increased substantially during the last decades, especially in New Zealand, Japan and United States [1]. The NRB uses alternate layers of natural rubbers and steel plates, it has small damping and often be used to accommodate the shrinkage of the deck of isolated bridges. In recent years, two evolving types of elastomeric bearings were invented, named as high damping rubber bearing (HDRB) and super high damping rubber bearing (SHDRB). The rubber material of HDRB and SHDRB possesses high damping due to the add of specific chemical fillers like carbon black, plasticizer and

44 oil during the vulcanization process. With these additives, HDRB and SHDRB provide structural systems additional damping to dissipate seismic energy and 
lengthen their fundamental period resulting in the decrease of the seismic response [2-3].

Since the acceptance of the performance-based design philosophy [4], nonlinear time history analysis is recommend by some design specifications [5-6] for the seismic response assessment of isolated bridges [7]. Hence, accurate modeling of the mechanical behavior of the elastomeric bearings is of great importance to a rational design of isolated bridges. In these specifications, the hysteretic behavior of NRB is approximated by equivalent linear method, and that of HDRB and SHDRB are represented by bilinear elasto-plastic model. However, the chemical fillers in rubber make the hysteretic behavior of the elastomeric bearings, in particular the HDRB and SHDRB, considerably complicated. Therefore, any existing linear models cannot reproduce their mechanical characteristics with satisfactory accuracy, especially the rate-dependent property as found by many researchers [8-9]. In the past studies, a few rate-dependent constitutive models for NRB and HDRB were proposed to ameliorate the aforementioned limitations [8-12], while the analytical models available to SHDRB are rare [3, 13]. Moreover, most of these theoretical models are based on the experimental hysteretic loops of the elastomeric bearings, this indicates the necessity for the further study of elastomeric bearings in terms of rubber material. On the other hand, NRB, HDRB and SHDRB are often used together in engineering practices, nowadays the design and seismic analysis of isolated bridges with elastomeric bearings are extremely complex because different hysteretic models should be employed, in this regard, a major need exists for a simplified analytical and design 
approach. Development of a generalized constitutive law to describe the mechanical behaviors of these three types of elastomeric bearings is motivated by these reasons.

In this paper, a generalized rate-dependent constitutive law for three types of

71 elastomeric bearings (i.e., NRB, HDRB, and SHDRB) under the horizontal shear

72 deformation is presented from the viewpoint of rubber material. A modified version of

73 classical Zener model is established to express the stress-strain function of rubber

74 material and the total stress is decomposed to rate-independent equilibrium stress and

75 rate-dependent overstress to describe the fundamental viscoelastic behavior of the

76 rubber. A nonlinear viscous damping coefficient is introduced into the mathematical

77 formulation to characterize the rate-dependent effect. Furthermore, multi-step

78 relaxation tests and monotonic shear tests are performed to identify the material

79 parameters. The numerical and experimental results are compared to verify the

80 accuracy of the proposed model. Finally, in order to investigate the accuracy and

81 feasibility of the proposed model on the application to the seismic response 82 assessment, real-time hybrid simulation (RTHS) test was performed and favorable 83 results were obtained.

\section{Strain energy function}

85 According to the phenomenological theory, rubber is a hyperelastic material and the mechanical properties of rubber can be characterized by its strain energy function $W$ [14], which can be represented in terms of deformation tensor invariants $\left(I_{1}, I_{2}, I_{3}\right)$,

$$
W=W\left(I_{1}, I_{2}, I_{3}\right)
$$

where 


$$
\left\{\begin{array}{l}
I_{1}=\operatorname{tr} \mathbf{B}=\lambda_{1}^{2}+\lambda_{2}^{2}+\lambda_{3}^{2} \\
I_{2}=\frac{1}{2}\left[(\operatorname{tr} \mathbf{B})^{2}-\left(\operatorname{tr} \mathbf{B}^{2}\right)\right]=\lambda_{1}^{2} \lambda_{2}^{2}+\lambda_{2}^{2} \lambda_{3}^{2}+\lambda_{3}^{2} \lambda_{3}^{2} \\
I_{3}=\operatorname{det} \mathbf{B}=\lambda_{1}^{2} \lambda_{2}^{2} \lambda_{3}^{2}
\end{array}\right.
$$

91 tr and det represent trace and determinant of left Cauchy-Green deformation tensor $\mathbf{B}$,

$92 \lambda_{i}(i=1,2,3)$ denotes principal stretches. Moreover, considering that rubber is 93 incompressible, the third invariant $I_{3}=1$, thus $W$ is expressed as a function of $I_{1}$ and $I_{2}$ 94 only, i.e., $W=W\left(I_{1}, I_{2}\right)$.

95 Since an adequate strain energy function is of great importance in establishing a reliable constitutive model, there are many strain energy function expressions in the literature [15], the most general one is proposed by Rivlin [16] as follows:

$$
W_{\text {Rivlin }}=\sum_{i=0, j=0}^{\infty} C_{i j}\left(I_{1}-3\right)^{i}\left(I_{2}-3\right)^{j}
$$

where $C_{i j}$ is the material parameter. expression and has been widely used for its simple form and sufficient accuracy to predict the response of rubber material from small to moderate strain amplitudes.

$$
W_{\text {Mooney-Rivlin }}=C_{10}\left(I_{1}-3\right)+C_{01}\left(I_{2}-3\right)
$$

104 where $C_{01}$ and $C_{10}$ are the material parameters.

105 However, strain energy functions available in published researches are mainly

106 focusing on rubber-like materials, the investigations on the behavior of innovative

107 rubber materials aforementioned are found only in a few works [12]. Furthermore,

108 previous researches mainly deals with the uni-axial stress-strain relationship of rubber material, hence, these studies fail to investigate the shear regime, which is essential 
110 for elastomeric bearings under the earthquake exaction. Therefore, development of an

111 adequate strain energy function applicable to shear deformation for seismic

112 application is aroused by these reasons.

113 Past investigations on the performances of existing strain energy functions showed

114 their incapability to represent the complex mechanical behaviors of hyperelastic

115 materials, especially the high stiffness at small strains [12]. This feature is referred to

116 as the Fletcher-Gent effect on the effect of fillers in NRB [18], it should be noted that

117 this effect seems more significant in HDRB and SHDRB due to the higher fillers

118 contents. In this study, in order to not only reproduce the rubber response accurately

119 but also minimize the material parameters and ensure the simplicity of mathematical

120 formulation, a strain energy function of $I_{1}$ and $I_{2}$ with an additional stiffness correction

121 factor $\alpha$ is proposed as follows:

$$
W\left(I_{1}, I_{2}\right)=C_{1}\left(I_{1}-3\right)+\frac{2}{3} C_{2}\left(I_{1}-3\right)^{\frac{3}{2}}+\frac{1}{2} C_{3}\left(I_{1}-3\right)^{2}+\frac{2}{5} C_{4}\left(I_{1}-3\right)^{\frac{5}{2}}+\frac{C_{5}}{\alpha+1}\left(I_{2}-3\right)^{\alpha+1}
$$

123 where $\mathrm{C}_{i}(i=1 \sim 5)$ and $\alpha$ are the material parameters.

\section{Constitutive equation for rate-dependent property}

125 To model the rate-dependent phenomenon of elastomeric bearings, a

126 phenomenological Zener model shown in Fig. 1 is considered. The hyperelastic spring

127 A represents the rate-independent equilibrium stress, while the Maxwell element

128 consisting of a hyperelastic spring $\mathrm{B}$ and a nonlinear dashpot $\mathrm{C}$ expresses the

129 rate-dependent overstress.

130 


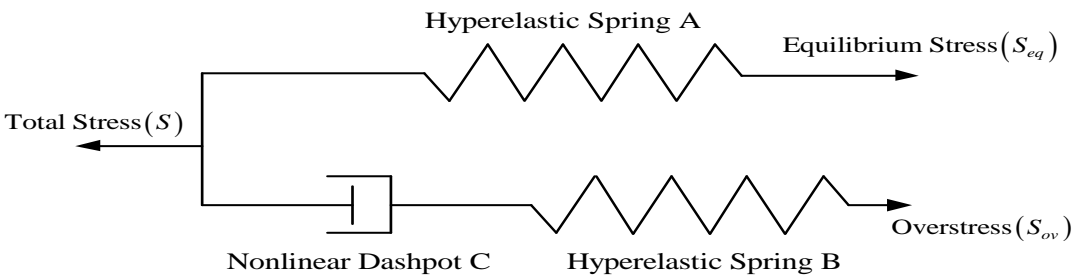

132

Fig. 1. Schematic of modified hyperelastic Zener model.

133 The total stress $\mathbf{S}$ is given by

$$
\mathbf{S}=\mathbf{S}_{e q}+\mathbf{S}_{o v}
$$

135 where $\mathbf{S}$ symbolizes the Cauchy stress, the subscript $e q$ and $o v$ denote equilibrium

136 stress and overstress, respectively. Furthermore, the Cauchy stress is expressed as

$$
\mathbf{S}=2\left(\mathbf{B} \frac{\partial W}{\partial I_{1}}-\mathbf{B}^{-1} \frac{\partial W}{\partial I_{2}}\right)-p \delta
$$

138 where $\delta$ is the Kronecker symbol, $p$ denotes the hydrostatic pressure determined by the

139 boundary condition, $\mathbf{B}$ is the left Cauchy-Green deformation tensor.

$140 \quad$ For shear deformation, we get

$$
\left\{\begin{array}{l}
\lambda_{2}=1 \\
\lambda_{3}=\lambda_{1}^{-1} \\
\gamma=\lambda_{1}-\lambda_{3}
\end{array}\right.
$$

142 where $\gamma$ is the simple shear strain.

143 substituting Eq. (8) into Eq. (2), $I_{1}$ and $I_{2}$ can be expressed as

$$
I_{1}=I_{2}=3+\gamma^{2}
$$

145 the left Cauchy-Green deformation tensor $\mathbf{B}$ and $\mathbf{B}^{-1}$ can be calculated as

$$
\mathbf{B}=\mathbf{F} \cdot \mathbf{F}^{T}=\left[\begin{array}{ccc}
1+\gamma^{2} & \gamma & 0 \\
\gamma & 1 & 0 \\
0 & 0 & 1
\end{array}\right] \text { and } \mathbf{B}^{-1}=\left[\begin{array}{ccc}
1 & -\gamma & 0 \\
-\gamma & 1+\gamma^{2} & 0 \\
0 & 0 & 1
\end{array}\right]
$$




$$
\mathbf{F}=\left[\begin{array}{lll}
1 & \gamma & 0 \\
0 & 1 & 0 \\
0 & 0 & 1
\end{array}\right]
$$

149 substituting Eqs. (9)-(11) into Eq. (7), the shear stress component is given by

$$
\mathbf{S}_{12}=2 \gamma\left(\frac{\partial W}{\partial I_{1}}+\frac{\partial W}{\partial I_{2}}\right)
$$

151 combing Eq. (5) and Eq. (12), one gets,

$$
\begin{aligned}
& \tau_{e q}=2\left(C_{1 e q} \gamma_{e q}+C_{2 e q} \gamma_{e q}^{2}+C_{3 e q} \gamma_{e q}^{3}+C_{4 e q} \gamma_{e q}^{4}+C_{5 e q} \gamma_{e q}^{2 \alpha_{e q}+1}\right) \\
& \tau_{o v}=2\left(C_{1 o v} \gamma_{o v}+C_{2 o v} \gamma_{o v}^{2}+C_{3 o v} \gamma_{o v}^{3}+C_{4 o v} \gamma_{o v}^{4}+C_{5 o v} \gamma_{o v}^{2 \alpha_{o v}+1}\right)
\end{aligned}
$$

154 where $C_{i e q}, C_{i o v}(i=1 \sim 5), \alpha_{e q}$ and $\alpha_{o v}$ are the material parameters determined from the 155 equilibrium response and instantaneous response, which corresponds to infinitely 156 slow and fast strain rate, respectively.

157 Based on a work by Huber and Tsakmakis [19], the rate of Left Cauchy-Green 158 deformation tensor $\mathbf{B}$ is defined by

$$
\dot{\mathbf{B}}_{B}=\mathbf{B}_{B} \mathbf{L}_{A}^{T}+\mathbf{L}_{A} \mathbf{B}_{B}-\frac{2}{\eta} \mathbf{B}_{B} \mathbf{S}_{B}{ }^{D}
$$

160 where $\eta$ is the viscosity parameter represented by the nonlinear dashpot element $\mathrm{C}$.

161 The $(\cdot)$ indicates material time derivative. Superscript $D$ denotes the deviatoric 162 component. $\mathbf{L}$ is the velocity gradient tensor given by

$$
\mathbf{L}=\dot{\mathbf{F}} \cdot \mathbf{F}^{-1}
$$

164 combining Eqs. (15)-(16), the expression of rate of $\mathbf{B}_{B}$ is obtained.

$$
\dot{\gamma}_{o v}=\dot{\gamma}_{e q}-\frac{4\left(C_{1 o v} \gamma_{o v}+C_{2 o v} \gamma_{o v}^{2}+C_{3 o v} \gamma_{o v}^{3}+C_{4 o v} \gamma_{o v}^{4}+C_{5 o v} \gamma_{o v}^{2 \alpha_{o v}+1}\right)}{\eta}\left(\frac{2}{3} \gamma_{o v}^{2}+1\right)
$$

166 when all the parameters are identified, the constitutive equation is developed. 


\section{Material tests and parameter identification}

168

169

170

171

172

173

176

177

178

179

180

181

182

183

184

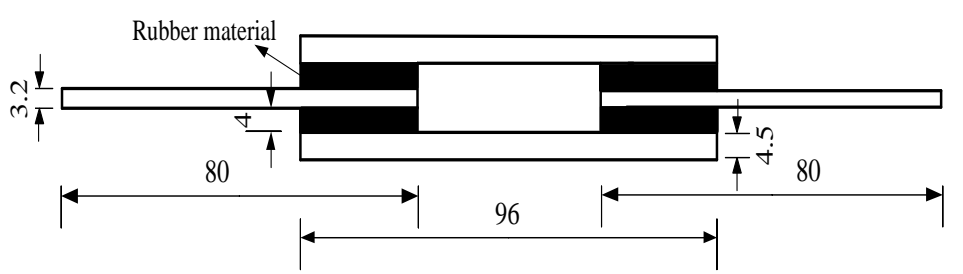

(a) Specimen (unit: $\mathrm{mm}$ )

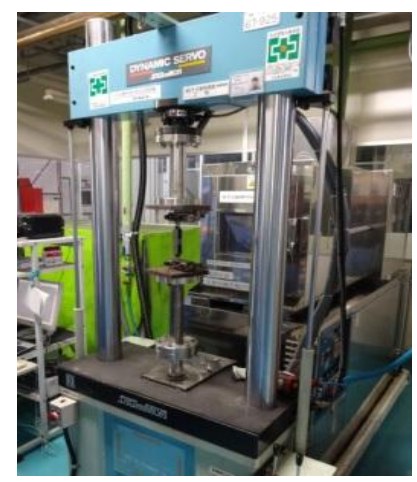

(b) Instrument

Fig. 2. Test specimen and instrument.

\subsection{Multi-step relaxation test and monotonic shear test}

A multi-step relaxation (MSR) tests was employed in the experimental scheme to obtain the equilibrium stress. In the tests, each specimen was tested up to the specified shear strains and held constant with a time interval of 20 minutes, Fig. 3 presents the applied strain history utilized in the test. 

to an almost constant value in all three types of rubber specimens, a similar characteristic of carbon black filled rubber was also observed by Lion [20]. By connecting all the minimum stress values at each corresponding strain level, the

194 equilibrium response can be achieved as shown in Fig. 5.

195

196

197

198

199

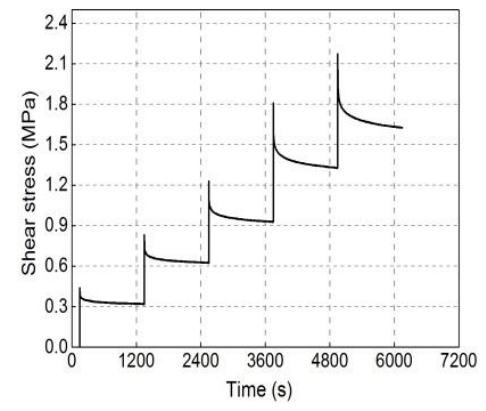

(a) NRB

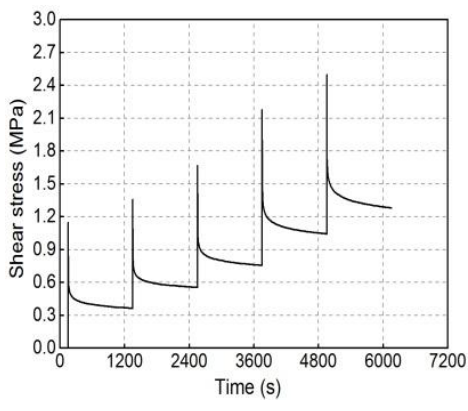

(b) HDRB

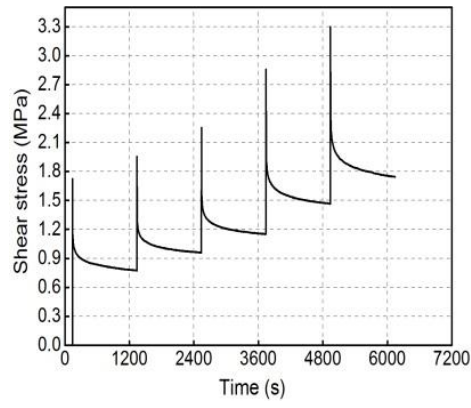

(c) SHDRB

Fig. 4. Stress time history obtained by MSR tests.

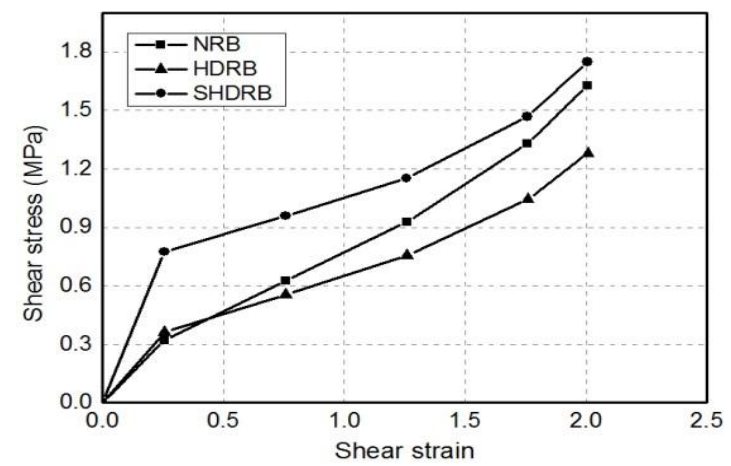

Fig. 5. Equilibrium response obtained by MSR tests. 
202 Theoretically, the instantaneous response can be ideally obtained when the rubber 203 material is loaded at an infinitely fast rate. However, the maximum stroke velocity of 204 the displacement controlled device inherently limits the loading rate of the specimen. 205 To overcome this limitation, a series of monotonic shear tests were conducted. The 206 tests were carried out at four different loading rates up to a maximum shear strain 207 range of 2.00. The four strain rates of $0.08,0.8,1.6$ and $4.01 / \mathrm{s}$ were utilized in the 208 tests. Fig. 6 shows the stress-strain response that were observed in four rate cases, in 209 general, the curves contained a threefold feature corresponding to small, moderate and 210 large strains, among which the extreme high initial stiffness at the small strain level is 211 the most prominent feature. Comparing the experimental data at different strain rates, 212 it was seen that all three types of elastomeric bearings possess a pronounced 213 rate-dependent behavior, especially in the case of HDRB and SHDRB. It should be 214 mentioned that the stress at the strain rate of $1.61 / \mathrm{s}$ is smaller than that of $0.81 / \mathrm{s}$ for 215 shear strain beyond about 1.25 for the HDRB, this abnormal phenomenon can be 216 mainly attributes to the experimental error in the tests and manufacturing error of 217 HDRB. A further comparison of the curves at different strain rates indicates a 218 diminishing trend, that at higher strain rate, the increase of the stress response was 219 declining. Similar phenomenon of HDRB was also observed in the cyclic shear tests 220 [10], in which the fastest strain rate was used to approach the neighborhood state of 221 the instantaneous response. For a similar consideration, the stress response obtained at $2224.01 / \mathrm{s}$ is regarded as an acceptable instantaneous response in this study. 


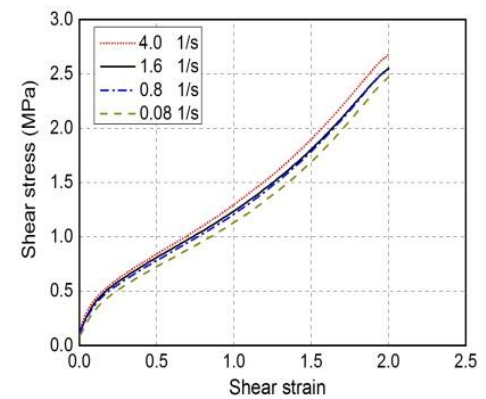

(a) NRB

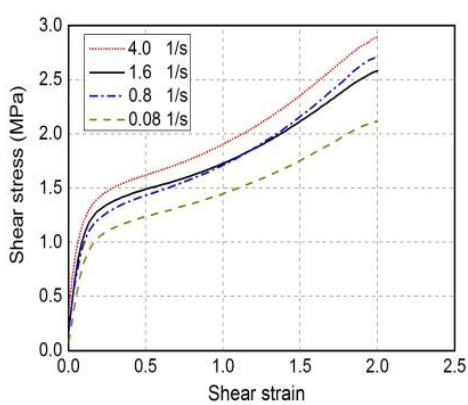

(b) HDRB

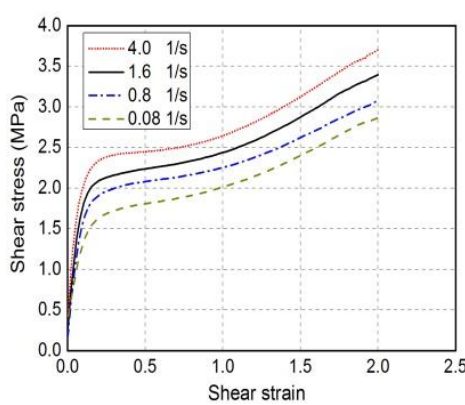

(c) SHDRB

Fig. 6. Stress-strain relationships obtained by monotonic shear tests.

\section{8}

229 technique in the small strain range wherein the high initial stiffness is dominant. Then, 242 other parameters $C_{i o v}(i=1 \sim 5)$ are identified by the least-square fitting method using

Table 1 Material parameters for equilibrium stress.

\begin{tabular}{cccccc}
\hline Specimen & $C_{1 e q}(\mathrm{MPa})$ & $C_{2 e q}(\mathrm{MPa})$ & $C_{3 e q}(\mathrm{MPa})$ & $C_{4 e q}(\mathrm{MPa})$ & $C_{5 e q}(\mathrm{MPa})$ \\
\hline NRB & 0.7642 & -0.7091 & 0.3883 & -0.0619 & 0 \\
HDRB & 1.0615 & -1.5318 & 0.9779 & -0.1994 & 0 \\
SHDRB & 2.0347 & -3.0981 & 1.9878 & -0.4239 & 0 \\
\hline
\end{tabular}

The overstress $\tau_{o v}$ is obtained by subtracting the equilibrium stress $\tau_{e q}$ from the total stress obtained by the monotonic shear tests using the case of $4.01 / \mathrm{s}$. Firstly, the parameter $\alpha_{o v}$ in Eq. (14) is determined by the shear stress-strain curve fitting 
243 the sets of the overstress $\tau_{o v}$ and the corresponding shear strain $\gamma_{o v}$ obtained from the

244 case of 4.0 1/s. The values of the overstress parameters are summarized in Table 2.

Table 2 Material parameters for overstress.

\begin{tabular}{ccccccc}
\hline Specimen & $C_{1 o v}(\mathrm{MPa})$ & $C_{2 o v}(\mathrm{MPa})$ & $C_{3 o v}(\mathrm{MPa})$ & $C_{4 o v}(\mathrm{MPa})$ & $C_{5 o v}(\mathrm{MPa})$ & $\alpha_{o v}$ \\
\hline NRB & 0.3561 & -0.4315 & 0.4666 & -0.1399 & 0.0141 & -0.35 \\
HDRB & 1.1306 & -1.0488 & 0.7869 & -0.2551 & 0.0264 & -0.35 \\
SHDRB & 1.7699 & -2.0761 & 1.7718 & -0.7801 & 0.1346 & -0.35 \\
\hline
\end{tabular}

248 The viscosity coefficient $\eta$ obtained from the tests results is found not to be

249 constant, showing variation within the progress of deformation of the specimen. In 250 this study, it is assumed that the viscosity coefficient has a functional relationship 251 with strain and strain rate, therefore, Gaussian function and a 3rd order polynomial 252 function were utilized to calculate viscosity coefficient, which provides a convenient 253 way to describe the strain and strain rate dependence of $\eta$.

$$
\eta=\left\{Z_{0}+A \exp \left[-0.5\left(\frac{\gamma-x_{c}}{w_{1}}\right)^{2}-0.5\left(\frac{v-y_{c}}{w_{2}}\right)^{2}\right]\right\} \times\left(B+C v+D v^{2}+E v^{3}\right)
$$

255 where $Z_{0}, A, x_{c}, w_{1}, y_{c} w_{2}, B, C, D$ and $E$ are material parameters, and $v$ is the strain 256 rate. The material parameters are determined by the nonlinear multivariate fitting 257 procedure, parameter results are listed in Table 3.

Table 3 Material parameters for dashpot element C.

\begin{tabular}{cccccccccc}
\hline$Z_{0}$ & $A$ & $x_{c}$ & $w_{1}$ & $y_{c}$ & $w_{2}$ & $B$ & $C$ & $D$ & $E$ \\
\hline 1.076 & 3.159 & 1.779 & 0.978 & 12.989 & 9.632 & 14.291 & -22.096 & 5.778 & 2.191 \\
\hline
\end{tabular}




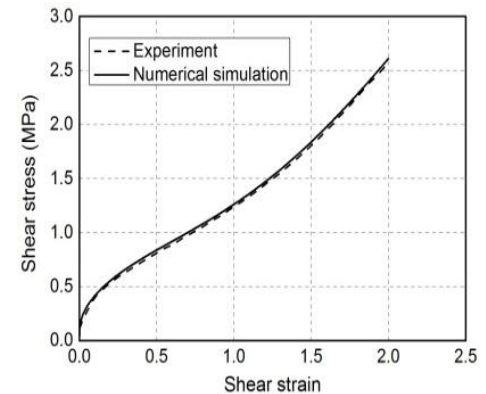

(a) Strain rate of $1.61 / \mathrm{s}$

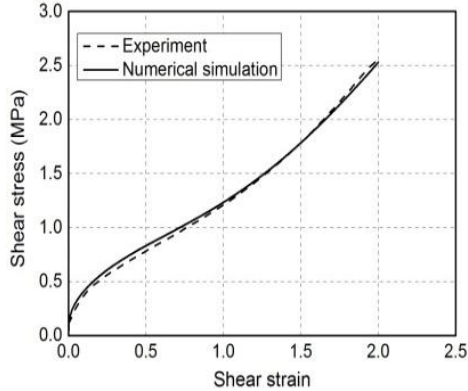

(b) Strain rate of $0.81 / \mathrm{s}$

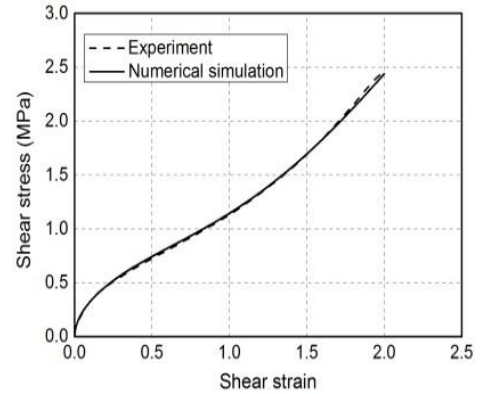

(c) Strain rate of $0.081 / \mathrm{s}$

Fig. 7. Numerical simulation and monotonic shear tests of NRB.

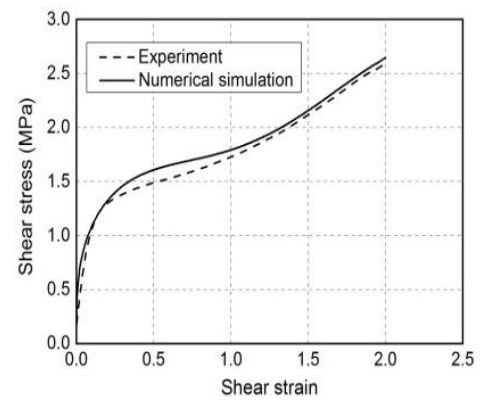

(a) Strain rate of $1.61 / \mathrm{s}$

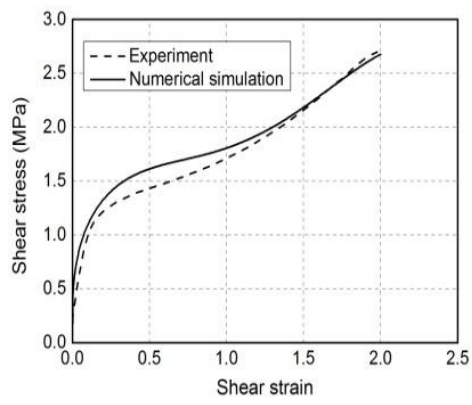

(b) Strain rate of $0.81 / \mathrm{s}$

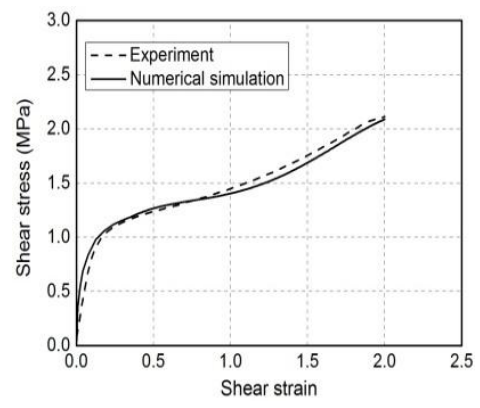

(c) Strain rate of $0.081 / \mathrm{s}$

Fig. 8. Numerical simulation and monotonic shear tests of HDRB.

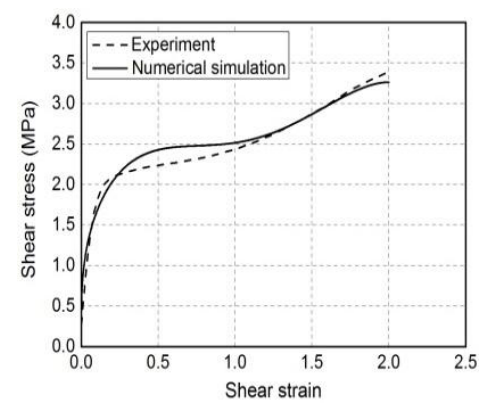

(a) Strain rate of $1.61 / \mathrm{s}$

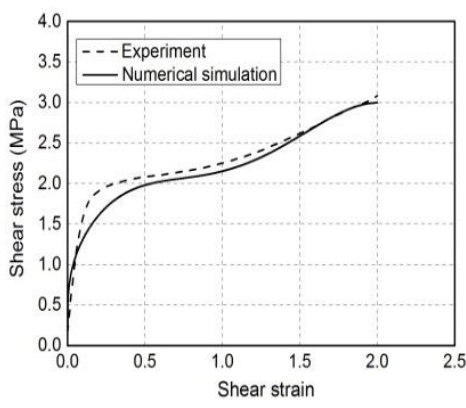

(b) Strain rate of $0.81 / \mathrm{s}$

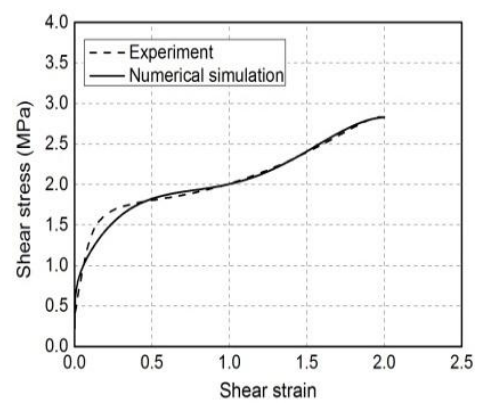

(c) Strain rate of $0.081 / \mathrm{s}$ 
281 In this section, the performance of seismic response assessment of the proposed 282 model is verified in comparison with the results of real-time hybrid simulation (RTHS) 283 test. In this test, an isolated single-column bridge as illustrated in Fig. 10 was exerted 284 by an accelerogram with a peak ground acceleration (PGA) of 207.8 gal. Using the 285 hybrid simulation technique, the bridge is split into numerical and experimental parts.

286 In this study, elastomeric bearings were tested physically to take advantage of RTHS 287 test in evaluating the dynamic response of rate-dependent devices and the remaining 288 components of the bridge was numerically simulated with sufficient accuracy. The 289 major challenging issue for RTHS test is to impose command displacement in 290 real-time, to overcome this problem, an innovative approach characterized by the 291 velocity-based method was adopted in this work, which improved the accuracy of 292 RTHS test results and simplified the test control algorithm [21].

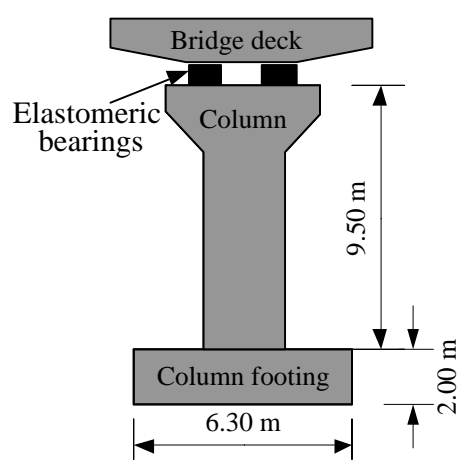
Fig. 10. Schematic view of a single-column bridge. nearly $10 \%$ and more distinct variations with a maximum error of almost $14 \%$ can be 
seen in the case of HDRB, coherent results were observed over the entire duration of

301 the earthquake excitations. It can be concluded that the displacement time histories in

302 three types of elastomeric bearings can be predicted accurately by the proposed model

303 and the model is accurate in the seismic response assessment of isolated bridge.

304

305

306

307

308

309

310

311

312

313

314

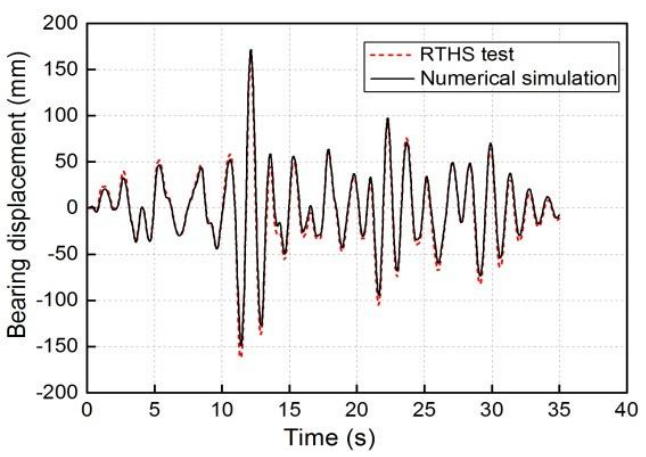

(a) NRB

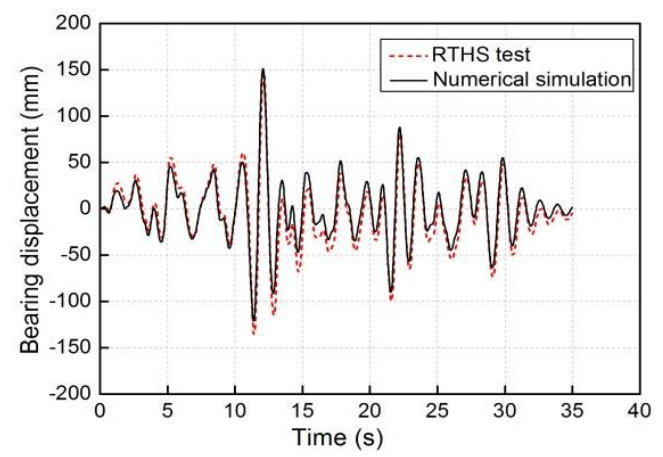

(b) HDRB

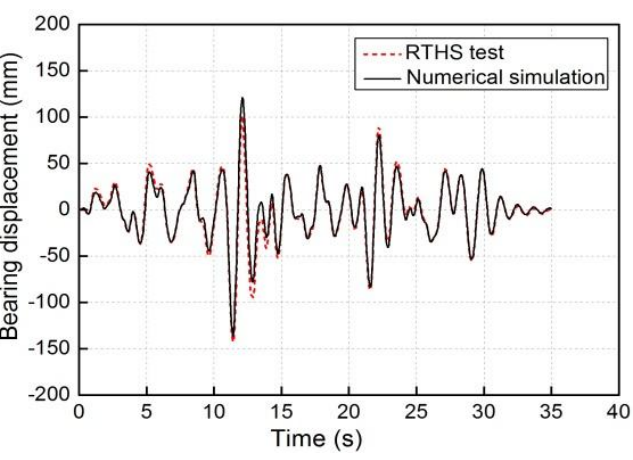

(a) SHDRB

Fig. 11. Comparisons of numerical simulation and RTHS test results.

\section{Conclusions}

This paper proposed a generalized mathematical expression to accurately represent the rate-dependent stress-strain relationship of three types of elastomeric bearings (i.e., NRB, HDRB and SHDRB). The conclusions are summarized as follows:

1. The newly developed model is a thermodynamically compatible rheological model including three elements to express the rate-dependent property of elastomeric 
318 bearings. Comparing the simulations using the proposed model with monotonic shear 319 tests and RTHS tests, it is concluded that the proposed model can reproduce the 320 rate-dependent behavior of elastomeric bearings with a good accuracy.

321 2. A novel strain energy function containing an additional stiffness correction factor 322 is proposed and it contributes to improve the description of high stiffness of 323 elastomeric bearings at small strains.

3243 . The proposed generalized model will be greatly facilitated and accelerated the 325 design and analysis process of isolated bridges. It can be utilized practically instead of 326 rigorous equivalent linear and bilinear models in predicting the stress-strain behavior 327 of elastomeric bearings, especially the HDRB and SHDRB which have prominent 328 nonlinear rate-dependent property.

\section{Acknowledgements}

330 The authors gratefully acknowledge the financial support from National Science 331 Foundation of China (No. 51278219) and National Basic Research Program of China 332 (973 Program, No. 2011CB013804).

\section{Reference}

334 [1] Jangid RS, Optimum lead-rubber isolation bearings for near-fault motions. Engineering $335 \quad$ Structures 2007; 29(10): 2503-2513.

336 [2] Abe M, Yoshida J, Fujino Y. Multiaxial behaviors of laminated rubber bearings and their 337 modelling. I: Experimental study. Journal of Structural Engineering 2004; 130(8):1119-1132.

338 [3] Iemura H, Igarashi A, Aoki T, Yamamoto Y. Real-time substructure hybrid earthquake loading 339 system for super high damping rubber bearings, 1 st International Conference on Advances in 340

341 [4] Usami T, Ge HB. A performance-based seismic design methodology for steel bridge systems. 342 Journal of Earthquake and Tsunami 2009; 3(3):175-93. 
343 [5] AASHTO. Guide specifications for seismic isolation design. 3rd ed. Washington, DC:

344 American Association of State Highway and Transportation Officials. 2010.

345 [6] Japan Road Association. Bearing support design guide for highway bridges. Japan; 2012 (in 346 Japanese).

347 [7] Filipov ET, Fahnestock LA, Steelman JS, Hajjar JF, LaFave JM, Foutch DA. Evaluation of 348 quasi-isolated seismic bridge behavior using nonlinear bearing models. Engineering $349 \quad$ Structures $2013 ; 49: 168-181$.

350 [8] Hwang JS, Ku SW. Analytical modeling of high damping rubber bearings. Journal of Structural Engineering, ASCE 1997; 123(8):1029-1036.

352 [9] Abe M, Yoshida J, Fujino Y. Multiaxial Behaviors of Laminated Rubber Bearings and Their Modeling. II: Modeling [J]. Journal of Structural Engineering 2004; 130(8): 1133-1144.

354 [10] Bhuiyan AR, Okui Y, Mitamura H, Imai T. A rheology model of high damping rubber bearings for seismic analysis: Identification of nonlinear viscosity. International Journal of Solids and Structures 2009; 46(7-8): 1778-1792.

[11] Kikuchi M, Aiken ID. An analytical hysteresis model for elastomeric seismic isolation bearings. Earthquake Engineering and Structural Dynamics 1997; 26:215-231.

359 [12] Bhuiyan AR, Ahmed E. Analytical expression for evaluating stress-deformation response of rubber layer under combined action of compression and shear. Construction and Building Materials 2007; 21:1860-1868.

[13] Yoshida J, Sugiyama T, and Fujino Y. Optimal bilinear models of super high damping rubber

[14] Khajehsaeid H, Arghavani J, Naghdabadi R. A hyperelastic constitutive model for rubber-like materials. European Journal of Mechanics A/Solids 2013; 38, 144-151.

367 [15] Aidy A, Hosseini M, Sahari BB. A review of constitutive models for rubber-like materials. American Journal of Engineering and Applied Sciences 2010; 3(1): 232-239.

[16] Rivlin RS. Large elastic deformations of isotropic materials IV. Further developments of the general theory. Philos Trans R Soc 1948; A241:379-397.

[17] Mooney M, A theory of large elastic deformation. Journal of Applied Physics 1940,11, $582-592$. 
373 [18] Amin AFMS, Wiraguna SI, Bhuiyan AR, Okui Y. Hyperelasticity model for Finite Element analysis of natural and high damping rubbers in compression and shear. Journal of 375 Engineering Mechanics, ASCE 2006; 132 (1): 1-11.

376 [19] Huber N, Tsakmakis C. Finite deformation viscoelasticity laws. Mechanics of Materials 2000, $377 \quad 32(1): 1-18$.

378 [20] Lion A. A constitutive model for carbon black filled rubber: experimental investigations and 379 mathematical representation. Continuum Mechanics and Thermodynamics 1996; 8:153-169.

380 [21] Yuan Y. Zhu HP. Isolated effection of the Xiazhang cross-sea isolated bridge. Journal of $381 \quad$ Earthquake and Tsunami 2011; 5(3): 297-307. 